\title{
ESCALA MULTIDIMENSIONAL NA AVALIAÇÃO DA DOR E SINTOMAS DE IDOSOS EM CUIDADOS PALIATIVOS
}

\author{
Jossiana Wilke Faller ${ }^{1}$, Adriana Zilly ${ }^{2}$, Cynthia Borges de Moura ${ }^{3}$, Pedro Henrique Brusnicki ${ }^{4}$
}

\begin{abstract}
RESUMO: Objetivou-se avaliar a dor e sintomas associados em idosos com câncer em cuidados paliativos em domicílio. Estudo quantitativo de corte transversal, realizado com 33 idosos, no município de Foz do Iguaçu, estado do Paraná, no período de janeiro a maio de 2015, por meio do Edmonton Symptom Assessment System e analisados pela estatística descritiva. Os idosos tinham a idade prevalente na sexta década de vida (60 a 69 anos), em sua maioria mulheres, casadas, com câncer de mama, baixa renda e baixa escolaridade, e portadoras de outras doenças associadas ao câncer. Verificou-se que $90,1 \%$ dos pacientes referem dor moderada, em queimação, diária e contínua no local acometido pelo tumor. Os sintomas associados à dor foram ansiedade, cansaço, depressão e redução do bem-estar. Conclui-se que é necessária a readequação das medidas para controle da dor e sintomas e maior investimento de gestores em saúde para possibilitar melhor assistência paliativa em domicílio.

DESCRITORES: Idosos; Cuidados paliativos; Manejo da dor; Assistência domiciliar; Enfermagem.
\end{abstract}

\section{MULTIDIMENSIONAL PAIN AND SYMPTOM ASSESSMENT SCALE FOR ELDERLY PEOPLE IN PALLIATIVE CARE}

ABSTRACT: The objective was to assess pain and associated symptoms in elderly cancer patients in home-based palliative care. Quantitative and cross-sectional study involving 33 elderly in Foz do Iguaçu, state of Paraná, Brazil, between January and May 2015, using theEdmonton Symptom Assessment System and analyzed using descriptive statistics. The prevalent age range of the elderly was between 60 and 69 years old, mostly women, married, suffering from breast cancer, low income and low education, with other diseases associated with cancer. It was verified that $90.1 \%$ of the patients mention daily and continuous moderate burning pain in the site affected by the tumor. The associated symptoms were anxiety, fatigue, depression and reduced wellbeing. Pain and symptom control measures need to be readjusted and health managers need to invest further in order to permit better palliative care at home. DESCRIPTORS: Elderly; Palliative care; Pain management; Home nursing; Nursing.

\section{ESCALA MULTIDIMENSIONAL DE EVALUACIÓN DEL DOLOR Y SÍNTOMAS EN ANCIANOS EN CUIDADOS PALIATIVOS}

RESUMEN: La finalidad fue evaluar el dolor y síntomas asociados en ancianos con cáncer en cuidados paliativos en domicilio. Estudio cuantitativo de cohorte trasversal, desarrollado con 22 ancianos en la ciudad de Foz do Iguaçu, estado de Paraná, Brasil desde enero hasta mayo del 2015 mediante el Edmonton Symptom Assessment System y analizados mediante estadística descriptiva. La edad prevalente de los ancianos era entre 60 y 69 años, la mayoría era mujer, casada, con cáncer de mama, baja renta y baja escolaridad, y portadoras de otras enfermedades asociadas al cáncer. Se verificó que $90.1 \%$ de los pacientes indican dolor moderado, en quemazón, diario y continuo en el sitio acometido por el tumor. Los síntomas asociados al dolor fueron ansiedad, cansancio, depresión y reducción del bienestar. Se concluye que es necesaria la readecuación de las medidas para control del dolor y síntomas y mayor inversión de gestores en salud para posibilitar mejor atención paliativa en domicilio.

DESCRIPTORES: Ancianos; Cuidados paliativos; Manejo del dolor; Atención domiciliaria de salud; Enfermería.

${ }^{1}$ Enfermeira. Doutoranda em Enfermagem. Docente de Enfermagem da Universidade Estadual do Oeste do Paraná. Foz do Iguaçu, PR, Brasil.

${ }^{2}$ Bióloga. Doutora em Ciências. Docente de Enfermagem e do Programa de Pós-Graduação Stricto Sensu em Ensino e em Saúde Pública em Região de Fronteira. Universidade Estadual do Oeste do Paraná. Foz do Iguaçu, PR, Brasil.

${ }^{3}$ Psicóloga. Doutora em Psicologia Clínica. Docente de Enfermagem e do Programa de Pós-Graduação Stricto Sensu em Ensino. Universidade Estadual do Oeste do Paraná. Foz do Iguaçu, PR, Brasil.

${ }^{4}$ Enfermeiro. Mestrando em Saúde Pública. Universidade Estadual do Oeste do Paraná. Foz do Iguaçu, PR, Brasil.

Autor Correspondente:

Jossiana Wilke Faller

Universidade Estadual do Oeste do Paraná

Av. Tarquinio Joslin dos Santos, 1300 - 85870-650 - Foz do Iguaçu, PR, Brasil

E-mail: jo_faller2015@usp.br
Recebido: 05/03/2016 Finalizado: 03/06/2016 


\section{INTRODUÇÃO}

O processo de envelhecimento tornou-se questão de saúde pública que repercute nas diferentes esferas da estrutura social, econômica, política e cultural, com demandas específicas e necessidades de mudanças nos diversos setores de atenção pública e privada ${ }^{(1)}$. É neste contexto que se revelam o aumento na prevalência de Doenças Crônicas Não Transmissíveis (DCNT), característica da transição epidemiológica no Brasil, intensificada a partir dos 60 anos, destacando-se as doenças osteoarticulares, a Hipertensão Arterial Sistêmica (HAS), as doenças cardiovasculares, o Diabetes Mellitus (DM), as doenças respiratórias crônicas, a doença cerebrovascular e o câncer. Deste modo, a demanda pelos serviços de saúde aumenta, haja visto que nos idosos as doenças são múltiplas e perduram por longo período de tempo, resultando em maior e mais prolongado uso desses serviços ${ }^{(2)}$.

A idade é um marcador de risco importante para todos os tipos de câncer, uma vez que tanto a incidência como a mortalidade aumentam exponencialmente após os 50 anos. Esse grupo etário exige atenção, já que, ao contrário dos pacientes mais jovens, o tratamento oncológico radical (cirurgia, associada ou não ao tratamento adjuvante e a radioterapia/quimioterapia) tem se mostrado discutível, uma vez que os sintomas do tratamento interferem significativamente na qualidade de vida $^{(3)}$.

Em relação ao tratamento, quando o paciente se encontra fora de possibilidades terapêuticas, fazem-se presente os Cuidados Paliativos (CPs), uma abordagem que aprimora a qualidade de vida do paciente e de seus familiares, por meio da prevenção e identificação precoce e alívio do sofrimento e da avaliação correta e tratamento da dor e demais problemas de ordem física, psicossocial e espiritual ${ }^{(4)}$.

Os CPs podem ser iniciados no ambiente hospitalar ou na atenção básica, nesse caso em situações clínicas estáveis, com doenças crônicas progressivas ou condições irreversíveis, que exijam cuidados de menor complexidade que no ambiente hospitalar ${ }^{(5)}$. $\mathrm{O}$ atendimento domiciliar contribui para otimização dos leitos hospitalares e reintegra o paciente ao núcleo familiar e de apoio. Com relação ao idoso, o atendimento domiciliar preserva ao máximo sua autonomia, buscando a recuperação de sua independência funcional ${ }^{(6)}$.

Dentre os inúmeros sintomas presentes nos indivíduos com câncer, a dor é causa de intenso sofrimento, incapacidade e prejuízos à qualidade de vida ${ }^{(7)}$. Por ser subjetiva e uma experiência individual, é de difícil avaliação e requer dos profissionais suporte educacional, conhecimento e instrumentos que contribuam para sua compreensão ${ }^{(8)}$. Um dos métodos utilizados para avaliar a dor é a Escala de Avaliação de Sintomas de Edmonton (ESAS), um instrumento que utiliza a combinação de sintomas físicos e psicológicos, composto por nove sintomas frequentemente encontrados em pacientes com câncer ${ }^{(7)}$.

Diante de ferramentas capazes de proporcionar um levantamento mais preciso da dor, e sendo este um sintoma muito frequente e causador de sofrimento intenso aos pacientes com câncer, e tendo em vista a importância dos CPs e o controle dos sintomas apresentados por idosos sem perspectivas de cura, o objetivo desse estudo foi avaliar a dor e sintomas associados em idosos com câncer em CPs em domicílio.

\section{- MÉTODO}

A pesquisa constituiu-se de um estudo quantitativo, de corte transversal, realizado junto a idosos com câncer de um município da Região Sul do Brasil, o qual possui uma população aproximada de 254 mil habitantes, destes, cerca de 7,2\% (18 mil) acima dos 60 anos de idade, abaixo da média nacional de 10,8\% ${ }^{(9)}$, assistidos por 28 Unidades Básicas de Saúde (UBS) e quatro unidades hospitalares.

Para os fins desta pesquisa, a população foi constituída por idosos que utilizam os serviços do Grupo Interdisciplinar de Suporte Oncológico (GISO), que oferece a prática paliativista em domicílio a pacientes acometidos pelo câncer sem perspectivas de cura. Para utilizar este serviço, o paciente deve ser encaminhado pelos profissionais do Centro Especializado em Oncologia, hospital referência no atendimento ao câncer para nove municípios circunvizinhos.

Integram o GISO profissionais da saúde de diferentes áreas, que realizam atendimento domiciliar por 
meio de visitas periódicas, com o objetivo de melhorar a qualidade de vida do paciente, considerando a família também como objeto de cuidado. Por ocasião da pesquisa, em janeiro de 2015, 717 usuários estavam cadastrados.

A amostra foi definida inicialmente por 150 idosos cadastrados no programa. Como critério de inclusão no estudo considerou-se ter 60 anos ou mais de idade, com capacidade para comunicar-se de forma verbal ou escrita e estar com dados atualizados no cadastro. Foram excluídos os que não se enquadravam nesses critérios. Para a coleta dos dados, desenvolvida durante os meses de janeiro a maio de 2015, obteve-se do serviço a ficha cadastral dos idosos para a identificação sociodemográfica e dados clínicos como localização do tumor, terapêutica e fármacos utilizados.

Após, procedeu-se contato via telefone com o paciente ou o cuidador/familiar a fim de convidálo a participar do estudo, bem como a aplicação de instrumento próprio tipo questionário que contemplava o perfil pessoal, socioeconômico e de acompanhamento à saúde. Foram excluídos nesta etapa da pesquisa os indivíduos que não possuíam contato telefônico ou dado incorreto; mudança de cidade; óbito; e casos em que não atendiam aos critérios de inclusão ou que se negaram a participar. Em casos de dificuldade de localização no domicílio, a ligação ou a visita (em caso de agendamento prévio), era repetida até três vezes, sendo o sujeito substituído na ordem sequencial da listagem. Ao final, a amostra foi constituída por 33 idosos.

Para avaliar a dor e sintomas associados, utilizou-se o instrumento Edmonton Symptom Assessment System (ESAS-r), versão revisada ${ }^{(10-11)}$. Trata-se de escala multidimensional que, por meio de combinação de sintomas físicos e psicológicos, como: dor, cansaço (falta de energia), sonolência (sentir-se com sono), náusea, apetite, falta de ar, depressão (sentir-se triste), ansiedade (sentir-se nervoso) e sensação de bem-estar, assegura uma avaliação sistemática.

O instrumento possui uma escala que varia de 0 a 10, na qual zero representa a ausência do sintoma e dez representa o sintoma em sua mais forte manifestação. Esta graduação ainda pode ser estratificada como de intensidade baixa $(<4)$, moderada $(\geq 4$ e $<7)$ e grave $(\geq 7)^{(12)}$. Recomenda-se que o doente avalie cada um dos sintomas de acordo com o que sente no preciso momento, e, diariamente, quando se encontram internados em instituições hospitalares ou lares. Para este estudo, o instrumento foi aplicado uma única vez, por não ser internamento domiciliar, mas acompanhamento. Para análise dos dados, estes foram registrados em planilhas e registrados em médias, mínima e máxima, por meio de estatística descritiva.

O estudo foi aprovado pelo Comitê de Ética em Pesquisa sob protocolo 861.927/2014.

\section{RESULTADOS}

Dos 33 indivíduos estudados, houve predomínio do sexo feminino 63,64\% (n=21), com a média de idade na sexta década de vida $63,64 \%(n=21)$, em sua maioria casada $51,52 \%(n=17)$, com baixa escolaridade $66,66 \%(n=22)$ e baixa renda familiar $93,94 \%(n=31)$. Embora $81,82 \%(n=27)$ fossem aposentados, $18,18 \%$ $(n=6)$ dependiam financeiramente da família, 39,39\% $(n=13)$ residiam com familiares, de modo que se apresentavam como seus principais cuidadores $42,42 \%(n=14)$, em sua maior parte com mais de 60 anos $45,46 \%(n=15)$.

A neoplasia de maior acometimento nas mulheres foi a de mama 27,27\% (nove) e no homem de base de língua 09,09\% (três). As demais neoplasias encontradas foram Colo do Útero, Linfoma Não Hodgkin, Reto, Esôfago, Exocervix, Laringe, Coluna Vertebral, Mieloma Múltiplo, Pulmão, Endométrio, Vagina, Melanoma, Nasofaringe, Pele, Próstata e Seio Piriforme, citadas em ordem de maior prevalência.

Nove idosos (27,27\%) estavam em tratamento antineoplásico e a terapia de maior prevalência foi a de associação $87,88 \%$ ( $n=29)$, na qual se utiliza radioterapia e quimioterapia associadas ou não a cirurgia. Além da medicina tradicional, nove sujeitos $(27,27 \%)$ relataram utilizar plantas medicinais para a busca da cura ou alívio de sintomas.

Em relação ao sono, 54,55\% ( $n=18)$ relataram menos de 8 horas/dia e sono interrompido 75,76\% $(n=25)$. Além do câncer, foram identificadas outras doenças crônicas associadas como HAS em nove $(27,27 \%)$. Oito $(24,24 \%)$ relataram que nos últimos 12 meses necessitaram de internamento por diversos 
motivos, incluindo a dor. Quanto à percepção da qualidade de vida, 75,76\% ( $\mathrm{n}=25)$ afirmaram como boa (Tabela 1).

Tabela 1 - Perfil sociodemográfico e clínico de idosos em Cuidados Paliativos em domicílio. Foz do Iguaçu, PR, Brasil, 2015

\begin{tabular}{|c|c|c|c|c|c|}
\hline Variáveis & $\mathbf{n}$ & $\%$ & Variáveis & $\mathbf{N}$ & $\%$ \\
\hline Sexo & & & Aposentados & & \\
\hline $\mathrm{F}$ & 21 & 63,64 & Sim & 27 & 81,82 \\
\hline$M$ & 12 & 36,36 & Não & 6 & 18,18 \\
\hline Faixa etária & & & Com quem vive & & \\
\hline 60 ト--- 70 & 21 & 63,64 & Familiares & 13 & 39,39 \\
\hline 70 ト--- 80 & 9 & 27,27 & Cônjuge & 11 & 33,33 \\
\hline 80 e mais & 3 & 9,09 & Só & 9 & 27,27 \\
\hline Estado Civil & & & Parentesco do cuidador & & \\
\hline Casado & 17 & 51,52 & Sem cuidador & 6 & 18,18 \\
\hline Divorciado & 7 & 21,21 & Cônjuge & 12 & 36,36 \\
\hline Viúvo & 6 & 18,18 & Familiares & 14 & 42,42 \\
\hline Solteiro & 3 & 9,09 & Amigo & 1 & 3,03 \\
\hline Escolaridade & & & Idade do cuidador & & \\
\hline Analfabeto & 6 & 18,18 & Sem cuidador & 6 & 18,18 \\
\hline$\leq 8$ anos & 22 & 66,66 & $<60$ anos & 12 & 36,36 \\
\hline$>8$ anos & 5 & 15,15 & $\geq 60$ anos & 15 & 45,46 \\
\hline Renda (SM) & & & Tratamento antineoplásico & & \\
\hline$<2$ & 31 & 93,94 & Sim & 9 & 27,27 \\
\hline$\geq 2$ & 2 & 6,06 & Não & 24 & 72,73 \\
\hline Localização do Tumor & & & Terapêutica & & \\
\hline Feminino & & & Associação & 29 & 87,88 \\
\hline Mama & 9 & 27,27 & Radioterapia/Quimioterapia & 3 & 9,09 \\
\hline Colo do útero & 2 & 6,06 & Cirurgia & 1 & 3,03 \\
\hline Outros & 10 & 30,3 & Outros métodos de tratamento & & \\
\hline Masculino & & & Plantas Medicinais & 9 & 27,27 \\
\hline Base de língua & 3 & 9,09 & Oração & 7 & 21,22 \\
\hline Amígdala & 1 & 3,03 & Medicina tradicional & 17 & 51,52 \\
\hline Outros & 8 & 24,24 & Horas de sono & & \\
\hline Outras doenças* & & & $<8$ horas & 18 & 54,55 \\
\hline Não & 16 & 48,49 & $\geq 8$ horas & 15 & 45,46 \\
\hline HAS & 9 & 27,27 & Tipo de sono & & \\
\hline Diabetes Mellitus & 5 & 15,15 & Interrompido & 25 & 75,76 \\
\hline Outras & 9 & 27,27 & Contínuo & 8 & 24,24 \\
\hline Internado últimos 12 meses & & & Qualidade de vida & & \\
\hline Sim & 8 & 24,24 & Muito Boa / Ótima & 2 & 6,06 \\
\hline \multirow[t]{2}{*}{ Não } & 25 & 75,76 & Boa & 25 & 75,76 \\
\hline & & & Ruim & 6 & 18,18 \\
\hline Total & 33 & 100 & Total & 33 & 100 \\
\hline
\end{tabular}

*A porcentagem desta categoria não equivale a 100\%, pois o mesmo indivíduo pode apresentar duas ou mais doenças.

Legenda: SM: Salário Mínimo (equivalente a $\mathrm{R} \$ 788,00$, vigente à época da coleta de dados); HAS: Hipertensão Arterial Sistêmica. 
Quanto à avaliação da dor e sintomas por meio do ESAS, a média dos valores demonstrou uma linha de tendência nos valores de média intensidade (4 ---7). Os sintomas com maior estratificação foram ansiedade, dor e cansaço, demonstrados na Figura 1. Outros sintomas referidos, que não continham na escala, foram prisão de ventre, diarreia, incontinência urinária e polaciúria, classificados como de média intensidade.

Quanto ao tipo, periodicidade e duração da dor, observa-se que 48,49\% ( $n=16)$ dos idosos descreveram a dor em "queimação", diária 60,61\% (n=20) e contínua 48,49\% (n=16) (Tabela 2).

Em relação ao uso de fármacos para o controle da dor e demais sintomas, os participantes citaram os analgésicos não opioides $69,70 \%(n=23)$, seguido dos opioides $45,45 \%(n=15)$, antiácidos e inibidores da secreção gástrica (ISG) $60,61 \%$ ( $n=20)$, além dos medicamentos para outras doenças como os antihipertensivos 45,5\% ( $\mathrm{n}=15)$ (Tabela 3).

Os demais fármacos, em ordem decrescente de frequência foram: diuréticos, hormônios tireoidianos, antianginosos e vasodilatadores, antiespasmódicos, ansiolíticos e hipnóticos, antineoplásicos, antibióticos, anticonvulsivantes, antilipidêmicos, antianêmicos, anticoagulantes, antieméticos e agentes procinérgicos, anti-histamínicos, antiosteoartrósicos, expectorantes, laxantes, multivitamínicos e relaxantes musculares.

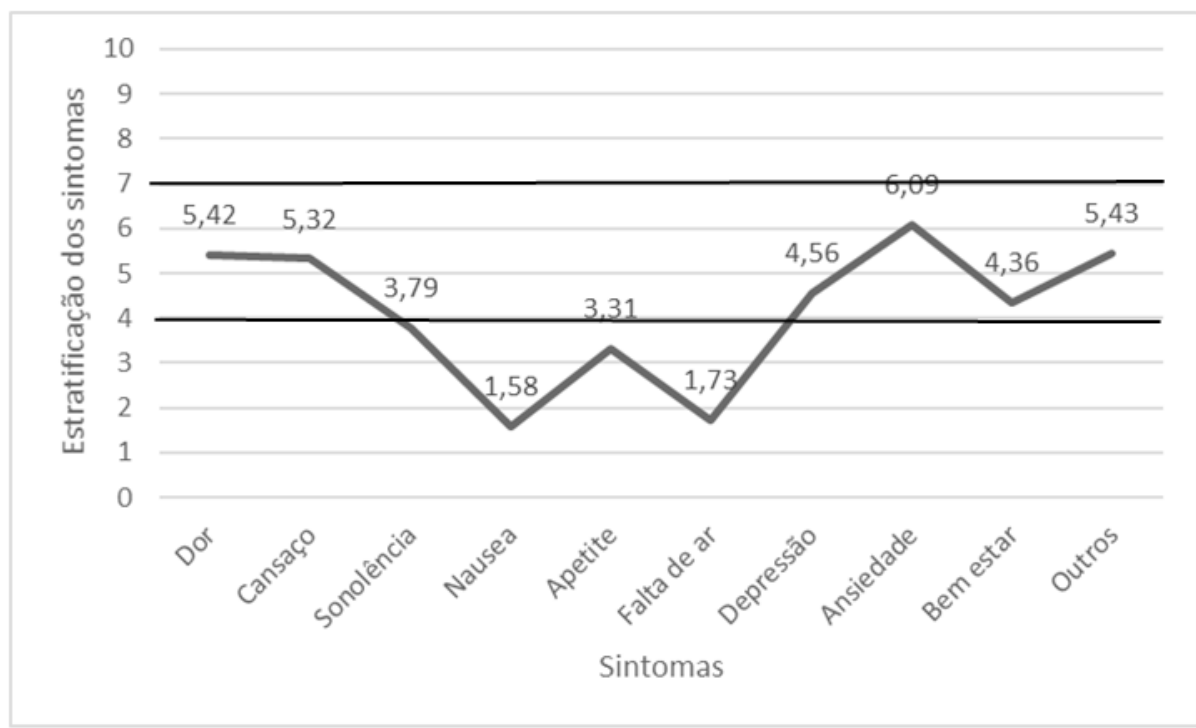

Figura 1 - Valores referentes aos sintomas na autoavaliação de idosos em Cuidados Paliativos, a partir do Edmonton Symptom Assessment System (ESAS-r). Foz do Iguaçu, PR, 2015

Tabela 2 - Caracterização da dor de idosos em Cuidados Paliativos em domicílio. Foz do Iguaçu, PR, Brasil, 2015

\begin{tabular}{lcclcc} 
Tipo da dor & $\mathbf{n}$ & $\mathbf{\%}$ & Periodicidade da dor & $\mathbf{n}$ & $\mathbf{\%}$ \\
\hline Queimação & 16 & 48,49 & Diariamente & 20 & 60,61 \\
\hline Pulsante & 5 & 15,15 & Dois dias ou mais & 10 & 30,3 \\
\hline Ausente & 3 & 9,09 & Ausente & 3 & 9,09 \\
\hline Cólica & 3 & 9,09 & Total & $\mathbf{3 3}$ & $\mathbf{1 0 0}$ \\
\hline Fisgada & 3 & 9,09 & Duração da dor & 16 & 48,49 \\
\hline Tração & 2 & 6,06 & Contínua & 14 & 42,42 \\
\hline Ardência & 1 & 3,03 & Intermitente & 3 & 9,09 \\
\cline { 3 - 6 } & & & Ausente & $\mathbf{3 3}$ & $\mathbf{1 0 0}$
\end{tabular}


Tabela 3 - Fármacos utilizados por idosos em Cuidados Paliativos em domicílio. Foz do Iguaçu, PR, Brasil, 2015

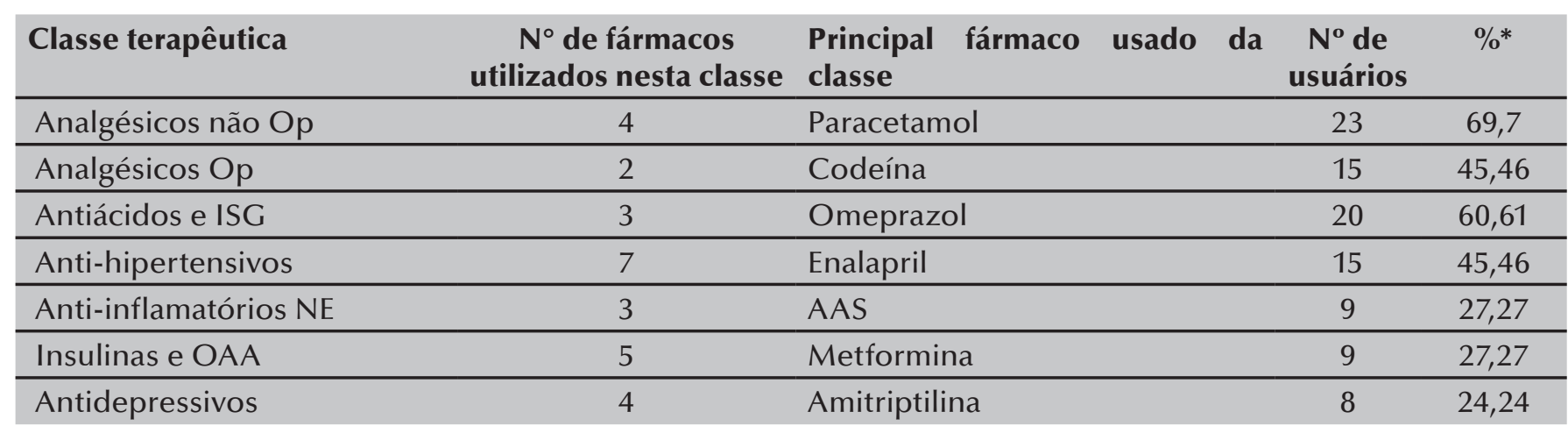

*A porcentagem dos fármacos está em relação ao número de usuários, os quais utilizam um ou mais, portanto não resulta em $100 \%$.

Legenda: Op.: Opioides; NE.: Não Esteroides; OAA: Outros Agentes Antidiabéticos. AAS: Ácido Acetilsalicílico.

\section{- DISCUSSÃO}

O ritmo de envelhecimento da população é observado por meio do Índice de Envelhecimento (IE), comparativamente entre áreas geográficas e grupos sociais. A região sul alcançou a condição de primeiro lugar em número proporcional de idosos $(13,6 \%)$ e o segundo lugar em expectativa de vida $(75,5 \text { anos })^{(1)}$. Junto a esse processo de envelhecimento populacional, ocorre o aumento de doenças crônicas, como o câncer, sendo a segunda causa de morte, perdendo apenas para as doenças cardiovasculares. Em relação ao gênero, sabe-se que ocorre no Brasil o processo de "feminização" do envelhecimento populacional, que é explicada pelos diferenciais de mortalidade entre homens e mulheres, tendendo a se ampliar nos grupos etários mais avançados ${ }^{(9)}$.

Corroborando com estes dados, esta pesquisa apontou como predominante o sexo feminino, com uma população de baixa renda e escolaridade e o câncer de mama como o de maior incidência. Segundo estudos, o envelhecimento da população feminina provocará uma elevação nas taxas de incidência do câncer de mama nos países de baixa e média renda ${ }^{(13)}$, justificada pela busca reduzida aos serviços de prevenção e detecção precoce ${ }^{(14)}$. No sexo masculino, o câncer de base de língua e assoalho bucal apresentou maior incidência, o qual predomina no sexo masculino, geralmente entre a quinta e sexta década de vida ${ }^{(15)}$.

Cerca $20 \%$ dos idosos ainda são dependentes financeiramente de familiares, sendo estes seus principais cuidadores. Considera-se que cuidar de idosos com câncer em situação que ameace a continuidade da vida torna-se um evento complexo no curso de vida familiar, pois além de enfrentar a sobrecarga do cuidado, lidam com o processo de aceitação e preparo do doente e dos demais familiares para o desfecho final da doença ${ }^{(16)}$.

Desta forma, buscam outros métodos de tratamento como o uso de plantas medicinais, em forma de chás, que provém de conhecimentos populares, e a aproximação com a espiritualidade, que facilita o enfrentamento da doença, diminuindo o sofrimento. As necessidades espirituais dos pacientes poderiam ser abordadas pelos profissionais de saúde e utilizadas como estratégia no planejamento da assistência ao paciente oncológico ${ }^{(17)}$.

Esses recursos foram utilizados junto à terapêutica médica, que em sua maioria foram de associação, ou seja, radioterapia, quimioterapia e/ou cirurgia. De acordo com o Instituto Nacional do Câncer (INCA), o protocolo terapêutico dependerá da localização do tumor e do seu estadiamento, assim como a idade, tamanho tumoral e grau de diferenciação celular, além da fase do diagnóstico ${ }^{(18)}$. Sobre a continuidade do tratamento antineoplásico em pacientes em CPs, que predominou no estudo (30\%), é uma proposta significativa e que interfere diretamente no tempo de sobrevida e na qualidade de vida dessas pessoas ${ }^{(19)}$.

Entre os efeitos da doença e do tratamento está a alteração do sono, descrita na literatura como 
frequente em pacientes com câncer. Houve número significativo de idosos em CPs que manifestaram sono interrompido e menos de 8 horas diárias. Distúrbios do sono, em especial a insônia, são frequentes em mulheres com câncer de mama, com prevalência maior do que na população em geral e relacionada mais com o diagnóstico da doença, uma condição aversiva que, juntamente com a dor, faz o indivíduo identificar fatores negativos de sua condição no período noturno ${ }^{(20)}$. Em pacientes oncológicos, a interrupção do sono, além de comprometer o funcionamento diurno, traz como consequência a fadiga e a depressão ${ }^{(21)}$.

O cansaço, ou fadiga, também apresentado no estudo com média moderada, é comum em idosos em tratamento oncológico. Outro estudo realizado com idosos com câncer identificou a fadiga em 60 idosos (42\%) em tratamento quimioterápico. É um sintoma que se desenvolve ao longo do tempo e tem implicações cognitivas, físicas e emocionais, incluindo o aumento da necessidade de repouso, redução da concentração mental e desinteresse em realizar atividades diárias ${ }^{(22)}$, afetando de forma negativa o bem-estar do indivíduo.

A ansiedade e a depressão, que se apresentaram de média intensidade, podem ter relação com a baixa escolaridade, baixo nível socioeconômico, enfermidades crônicas e incapacitantes, abandono e isolamento social, além da falta de atividade física ${ }^{(23)}$. Além disso, é comum no processo de envelhecimento a manifestação de doenças crônicas concomitantes, o que agrava os sintomas e requer maior cuidado no manejo da doença. A necessidade de internação, relatada pelos idosos do estudo, deve incluir uma avaliação minuciosa da causa, pois envolve fatores como interação medicamentosa, infecção e alteração metabólica ${ }^{(24)}$.

Em relação à dor, este é o sintoma mais frequente, manifestado em 90,1\% $(n=30)$ dos idosos da pesquisa. Aos sintomas álgicos somam-se às incapacidades relacionadas à neoplasia e seu tratamento, que podem ocasionar insônia, anorexia, confinamento ao leito, perda do convívio social, redução das atividades profissionais e de lazer ${ }^{(25)}$. A dor acarreta estresse e sofrimento não só aos doentes, mas também aos familiares/cuidadores, e pode resultar em alterações emocionais, sociais e econômicas desfavoráveis a todos.

Em relação aos fármacos utilizados, os analgésicos opioides como morfina e codeína, analgésicos não opioides como paracetamol e dipirona e o antidepressivo amitriptilina são indicados pelo Ministério da Saúde para o controle da dor ${ }^{(26)}$. Vale salientar que o tratamento da dor pode ser realizado por meio farmacológico (analgésicos não opioides, analgésicos opioides e adjuvantes), não farmacológicos (intervenção psicológica, física ou radioterapia) e invasivos (analgesia intratecal, bloqueio nervoso, cirurgia paliativa, neurocirurgia). Ocorrências da dor são observadas em pacientes em todo o mundo, acometendo até $80 \%$ dos pacientes durante os estágios do câncer, sendo que metade dos casos descrevem-na como moderada e $30 \%$ como insuportável ${ }^{(27)}$.

Ao observar os principais fármacos presentes no controle de sintomas destes idosos, é perceptível a necessidade de reavaliação para readequação da farmacoterapia, uma vez que a presença da dor e sua intensidade nesses indivíduos são representativas, associando às intervenções da área nutricional, psicológica e fisioterápica, de modo a proporcionar uma melhora na qualidade de vida dessa população.

Vale salientar que existem modalidades terapêuticas complementares disponíveis, como a musicoterapia, a massagem e a acupuntura para o controle da dor e outros sintomas, que beneficiam a qualidade de vida, promoção do relaxamento e do prazer, bem como fortalecimento do vínculo entre paciente/família/profissional(28).

\section{- CONCLUSÃO}

O estudo teve como objetivo avaliar a dor e demais sintomas presentes em pacientes idosos em cuidados paliativos acometidos pelo câncer. O perfil dos idosos participantes foi de prevalência do sexo feminino, na sexta década de vida e de baixa escolaridade. As neoplasias de maior acometimento na mulher foi a de mama, e no homem, de base da língua, com terapêutica de associação. A avaliação dos sintomas apontou que $90,1 \%$ dos indivíduos referem dor no local acometido pelo tumor, do tipo em "queimação", que se apresenta de forma contínua e de frequência diária. 
A ansiedade, o cansaço, a depressão e sensação de bem-estar/mal-estar manifestam-se como de intensidade moderada a severa, além de prisão de ventre, diarreia, incontinência urinária e polaciúria. Esses sintomas, quando associados, geram desconforto no idoso diminuindo sua qualidade de vida e motivação para as atividades físicas, sociais e de lazer.

Diante dos dados, identifica-se a necessidade de readequação das medidas de controle da dor e sintomas dos pacientes, pois geram impacto na qualidade de vida dos indivíduos. No Brasil, os CPs ainda não estão estruturados adequadamente, embora tenham apresentado um crescimento significativo a partir do ano 2000, com a consolidação de alguns serviços e a criação de outros. Novas iniciativas estão surgindo, mas o trabalho ainda é desafiador.

Realizar a coleta de dados no domicílio permitiu a observação da realidade vivenciada pelos idosos e seus familiares, como a condição física e emocional. A carência por orientações básicas referentes à doença, controle de sintomas e medidas de conforto foi percebida, evidenciando o importante papel da equipe de saúde, pois sabe-se que grande parte da ansiedade se dá pelo medo ou insegurança frente ao desconhecido.

Os baixos recursos humanos, financeiros e tecnológicos oferecidos pelo município tornam o trabalho dos profissionais paliativistas solitário e desgastante. $O$ investimento em capacitação dos profissionais na atenção básica é também um meio consistente de suprir as necessidades desses pacientes, visto que estes se encontram no domicílio, próximo às equipes de saúde da família ou unidades básicas de saúde.

O baixo investimento na educação continuada dos profissionais, e a não contratação de recursos humanos destinados aos CPs, contribuem para a não efetivação das políticas e programas destinados à atenção em saúde desses idosos, visto a complexidade e cronicidade que envolve o cuidar do idoso em CPs.

Para trabalhos futuros, sugere-se aplicar a escala em mais de um momento, ao mesmo doente, pois como limitação do estudo está à escassez de tempo e de recursos, limitando a pesquisa para análises comparativas, a respeito da dor e suas associações.

\section{REFERÊNCIAS}

1. da Luz EP, Dallepiane LB, Kirchner RM, da Silva LAA, da Silva FP, Kohler J, et al. Perfil sociodemográfico e de hábitos de vida da população idosa de um município da região norte do Rio Grande do Sul, Brasil. Rev. bras. geriatr. gerontol. [Internet] 2014; 17(2) [acesso em 04 jul 2015]. Disponível: http://dx.doi.org/10.1590/S1809-98232014000200008.

2. Campolina AG, Adami F, Santos JLF, Lebrão ML. A transição de saúde e as mudanças na expectativa de vida saudável da população idosa: possíveis impactos da prevenção de doenças crônicas. Cad. Saúde Publica. [Internet] 2013; 29(6) [acesso em 05 jul 2015]. Disponível: http://dx.doi.org/10.1590/S0102-311X2013000600018.

3. Toneti BF, de Paula JM, Nicolussi AC, Sawada NO. Qualidade de vida relacionada à saúde de idosos com câncer em tratamento adjuvante. Rev Rene. [Internet] 2014; 15(6) [acesso em 25 jun 2015]. Disponível:

http://dx.doi.org/10.15253/2175-6783.2014000600017.

4. de Andrade CG, da Costa SFG, Lopes MEL. Cuidados paliativos: a comunicação como estratégia de cuidado para o paciente em fase terminal. Ciênc. saúde coletiva. [Internet] 2013; 18(9) [acesso em 25 jun 2015]. Disponível: http://dx.doi.org/10.1590/S1413-81232013000900006.

5. Silveira MH, Ciampone MHT, Gutierrez BAO. Percepção da equipe multiprofissional sobre cuidados paliativos. Rev. bras. geriatr. gerontol. [Internet] 2014; 17(1) [acesso em 24 jun 2015]. Disponível:

http://dx.doi.org/10.1590/S1809-98232014000100002.

6. Fripp JC. Ação prática do paliativista na continuidade dos cuidados em domicílio. In: de Carvalho RT, Parsons HA, organizadores. Manual de Cuidados Paliativos ANCP. Porto Alegre: Sulina; 2012. p. 375-91.

7. Monteiro DR, Kruse MHL, Almeida MA. Avaliação do instrumento Edmonton Symptom Assessment System em cuidados paliativos: revisão integrativa. Rev. Gaúcha Enferm. [Internet] 2010; 31(4) [acesso em 20 nov 2014]. 
8. Waterkemper R, Reibnitz KS, Monticelli M. Dialogando com enfermeiras sobre a avaliação da dor oncológica do paciente sob cuidados paliativos. Rev. bras. enferm. [Internet] 2010; 63(2) [acesso em 20 jan 2015]. Disponível: http://dx.doi.org/10.1590/S0034-71672010000200026.

9. Instituto Brasileiro de Geografia e Estatística (IBGE). Primeiros resultados definitivos do Censo de 2010. BrasíliaDF: Ministério do Planejamento, Orçamento e Gestão [Internet] 2014 [acesso em 25 set 2015]. Disponível: http://www.ibge.gov.br/home/estatistica/populacao/censo2010/.

10. Bruera E, Kuehn N, Miller MJ, Selmser P, Macmillan K. The Edmonton Symptom Assessment System (ESAS): a simple method for the assessment of palliative care patients. J Palliat Care. [Internet] 1991; 7(2): 6-9.

11. Monteiro DR, Almeida MA, Kruse MHL. Tradução e adaptação transcultural do instrumento Edmonton Symptom Assessment System para uso em cuidados paliativos. Rev. Gaúcha Enferm. [Internet] 2013; 34(2) [acesso em 20 nov 2015]. Disponível: http://dx.doi.org/10.1590/S1983-14472013000200021.

12. Ribeiro ASS. Controlo de sintomas em cuidados paliativos num serviço de medicina interna [dissertação]. Portugal (Lisboa): Universidade de Lisboa/ Faculdade de Medicina; 2012.

13. da Silva RCF, Hortale VA. Rastreamento do câncer de mama no Brasil: quem, como e por quê? Rev. Bras. Cancerol. [Internet] 2012; 58(1) [acesso em 22 nov 2015]. Disponível: http://www1.inca.gov.br/rbc/n_58/v01/ pdf/10b_artigo_opiniao_rastreamento_cancer_mama_brasil_quem_como_por_que.pdf.

14. Höfelmann DA, dos Anjos JC, Ayala AL. Sobrevida em dez anos e fatores prognósticos em mulheres com câncer de mama em Joinville, Santa Catarina, Brasil. Ciênc. saúde coletiva. [Internet] 2014; 19(6) [acesso em 20 nov 2015]. Disponível: http://dx.doi.org/10.1590/1413-81232014196.03062013.

15. Bonfante GMS, Machado CJ, de Souza PEA, Andrade EIG, Acurcio FA, Cherchiglia ML. Sobrevida de cinco anos e fatores associados ao câncer de boca para pacientes em tratamento oncológico ambulatorial pelo Sistema Único de Saúde, Brasil. Cad. Saúde Publica. [Internet] 2014; 30(5) [acesso em 20 nov 2015]. Disponível:

http://dx.doi.org/10.1590/0102-311X00182712.

16. Matos E, de Pires DEP, Campos GWS. Relações de trabalho em equipes interdisciplinares: contribuições para novas formas de organização do trabalho em saúde. Rev. bras. enferm. [Internet] 2009; 62(6) [acesso em 22 nov 2015]. Disponível: http://dx.doi.org/10.1590/S0034-71672009000600010.

17. Guerrero GP, Zago MMF, Sawada NO, Pinto MH. Relação entre espiritualidade e câncer: perspectiva do paciente. Rev. bras. enferm. [Internet] 2011; 64(1) [acesso em 22 nov 2015]. Disponível:

http://dx.doi.org/10.1590/S0034-71672011000100008.

18. Brasil. Instituto Nacional de Câncer José Alencar Gomes da Silva (INCA). Tratamento do Câncer. Rio de Janeiro: INCA; 2015 [acesso em 20 jul 2015]. Disponível:

http://www2.inca.gov.br/wps/wcm/connect/cancer/site/tratamento.

19. da Silva MM, da Silva JA, Esteves LO, Mesquita MGR, Stipp MAC, Duarte SCM. Perfil sociodemográfico e clínico de pessoas em tratamento quimioterápico: subsídios para o gerenciamento em enfermagem. Rev. Eletr. Enf. [Internet] 2013; 15(3) [acesso em 10 fev 2016]. Disponível: http://dx.doi.org/10.5216/ree.v15i3.18417.

20. Rafihi-Ferreira RE, Soares MRZ. Insônia em pacientes com câncer de mama. Estud. psicol. [Internet] 2012; 29(4) [acesso em 20 abr 2015]. Disponível: http://dx.doi.org/10.1590/S0103-166X2012000400014.

21. Mansano-Schlosser TC, Ceolim MF. Factors associated with sleep quality in the elderly receiving chemotherapy. Rev. Latino-Am. Enfermagem. [Internet] 2012; 20(6) [acesso em 20 jan 2016]. Disponível: http://dx.doi.org/10.1590/S0104-11692012000600012.

22. Mansano-Schlosser TC, Ceolim MF. Fadiga em idosos em tratamento quimioterápico. Rev. bras. enferm. [Internet] 2014; 67(4) [acesso em 02 fev 2016]. Disponível: http://dx.doi.org/10.1590/0034-7167.2014670419.

23. Minghelli B, Tomé B, Nunes C, Neves A, Simões C. Comparison of levels of anxiety and depression among active and sedentary elderly. Rev. psiquiatr. clín. [Internet] 2013; 40(2) [acesso em 02 fev 2016]. Disponível: http://dx.doi.org/10.1590/S0101-60832013000200004. 
24. Brasil. Instituto Nacional de Câncer José Alencar Gomes da Silva (INCA). Controle de sintomas do câncer avançado em adultos. Normas e recomendações do INCA/MS. Rev. Bras. Cancerol. [Internet] 2000; 46(3) [acesso em 23 fev 2016]. Disponível: http://www.inca.gov.br/rbc/n_46/v03/pdf/normas.pdf.

25. Cardoso AICR. Controlo da dor em pacientes oncológicos [dissertação]. Portugal (Porto): Universidade do Porto. Instituto de Ciências Biomédicas Abel Salazar; 2014.

26. Ministério da Saúde (BR). Secretaria de Atenção à Saúde. Departamento de Atenção Básica. Caderno de atenção domiciliar. Brasília: Ministério da Saúde; 2013.

27. Barbosa JAA, Belém LF, Sette IMF, Carmo ES, Pereira GJS, da Silva Júnior ED. Farmacoterapia adjuvante no tratamento da dor oncológica. Rev. Bras. Promoc. Saude. [Internet] 2008; 21(2) [acesso em 23 fev 2016]. Disponível: http://www.redalyc.org/articulo.oa?id=40811362006.

28. Caires JS, Andrade TA, do Amaral JB, Calasans MTA, Rocha MDS. A utilização das terapias complementares nos cuidados paliativos: benefícios e finalidades. Cogitare Enferm. [Internet] 2014; 19(3) [acesso em 23 fev 2016]. Disponível: http://dx.doi.org/10.5380/ce.v19i3.33861. 